\title{
4d Lorentzian Holst action with topological terms
}

\author{
Danilo Jimenez Rezende and Alejandro Perez \\ Centre de Physique Théorique*, Campus de Luminy, 13288 Marseille, France.
}

(Dated: November 5, 2018)

\begin{abstract}
We study the Hamiltonian formulation of the general first order action of general relativity compatible with local Lorentz invariance and background independence. The most general simplectic structure (compatible with diffeomorphism invariance and local Lorentz transformations) is obtained by adding to the Holst action the Pontriagin, Euler and Nieh-Yan invariants with independent coupling constants. We perform a detailed canonical analysis of this general formulation (in the time gauge) exploring the structure of the phase space in terms of connection variables. We explain the relationship of these topological terms, and the effect of large $S U(2)$ gauge transformations in quantum theories of gravity defined in terms of the Ashtekar-Barbero connection.
\end{abstract}

\section{INTRODUCTION}

The possibility of describing the phase space of gravity as a background independent $S U(2)$ connection gauge theory is a remarkable property of the first order formulation of general relativity in four dimensions. This is the basis of the canonical quantization program of gravity known as loop quantum gravity [1]. After the discovery of the self dual connection formulation of canonical general relativity by Ashtekar [2], it was soon realized by Barbero [3] that a formulation in terms of a real $S U(2)$ connection was indeed possible. The only price to be paid is the appearance of a new free parameter $\gamma \in \mathbb{R}-\{0\}$ (the so-called Immirzi parameter [4]) into the definition of the canonical variables. A first step in clarifying the origin of the Immirzi parameter was to show [5] that the Ashtekar-Barbero variables can be obtained directly from the Hamiltonian formulation of general relativity defined by the first order action

$$
S[e, \omega]=\int \star\left(e^{I} \wedge e^{J}\right) \wedge F_{J I}(\omega)+\frac{1}{\gamma} e^{I} \wedge e^{J} \wedge F_{I J}(\omega)
$$

where $e$ is a vierbein and $\omega$ is a Lorentz connection. The first term is the standard Palatini action of general relativity, while second term can be shown not to affect the classical equations of motion. The reason for this is that $\delta_{\omega} S=0$ is independent of $\gamma$, and implies the connection to be the uniquely defined torsion free connection compatible with $e: \omega=\omega(e)$. The second term contribution to the equation $\delta_{e} S=0$ vanishes identically when evaluated on $\omega(e)$ due to the Riemann tensor identity $R_{[a b c] d}=0$.

The canonical formulation of the Holst action leads in this way to a one parameter family of $S U(2)$ connection formulations of the phase space of general relativity: all of them related by canonical transformations. However, in the quantum theory the canonical transformations relating different connection formulations appear not to be unitarily implemented. For instance the spectra of geometric operators are modulated by $\gamma$. Formally speaking, the off shell contributions of the second term in the action (10) have a non trivial effect on amplitudes in the path integral formulation of quantum gravity.

There is at least another real parameter-describing the family of possible $S U(2)$ connection formulations of gravity - with very similar qualitative effects: the so called $\theta$ parameter. Again, this parameter labels classically equivalent formulations that become physically different upon quantization. The reason for this is geometrically more transparent than the case of the Immirzi

\footnotetext{
* Unité Mixte de Recherche (UMR 6207) du CNRS et des Universités Aix-Marseille I, Aix-Marseille II, et du Sud Toulon-Var; laboratoire afilié à la FRUMAM (FR 2291)
} 
parameter as the effects of the $\theta$ parameter in the quantum theory are associated to the transformation properties of physical states under large $S U(2)$ gauge transformations [12, 13]. Physically, the phenomenon is in strict analogy with the $\theta$ parameter effects in QCD.

All this motivates the following questions (we shall explore in this work): Are there yet more general $S U(2)$ connection formulations of gravity? i.e., are there new parameters in addition to $\gamma$ and $\theta$ ? and if so, how naturally they arise from the Lagrangian framework, and, what are their possible physical effects upon quantization? More particularly, does the $\theta$ parameter in the connection formulation of gravity have a natural description at the Lagrangian level? We will shed some light on these questions by studying the canonical formulation of a general family of actions for general relativity (in a sense described below).

Holst's action allows to understand the presence of a non-vanishing and finite Immirzi parameter from a more clear standpoint. In fact, not having the second term in the first order formulation of general relativity (i.e. choosing $\gamma=\infty$ or in other words the Palatini formulation) would be un-natural from the Wilsonian perspective that calls for including in the action principle all terms compatible with the symmetry and field content of the theory. From this perspective the Immirzi parameter $\gamma \in \mathbb{R}-\{0\}$ is not an input but a consequence of local Lorentz plus diffeomorphism invariance together with the choice of $e$ and $\omega$ as fundamental fields. If we pursue this logic further then the most general action principle - compatible with diffeomorphism invariance and Lorentz invariance - describing pure-gravity in the first order formalism is

$$
\begin{aligned}
S[e, \omega] & =\int \overbrace{\alpha_{1} \star\left(e^{I} \wedge e^{J}\right) \wedge F_{J I}(\omega)+\alpha_{2} e^{I} \wedge e^{J} \wedge F_{I J}(\omega)}^{\text {Holst }}+\overbrace{\alpha_{3} F^{I J}(\omega) \wedge F_{J I}(\omega)}^{\text {Pontrjagin }} \\
& +\underbrace{\alpha_{4} F^{I J}(\omega) \wedge \star F_{J I}(\omega)}_{\text {Euler }}+\alpha_{5} \underbrace{d_{\omega} e^{I} \wedge d_{\omega} e_{I}-e^{I} \wedge e^{J} \wedge F_{I J}(\omega)}_{\text {Nieh }-Y \text { an }} \\
& +\alpha_{6} \underbrace{\underbrace{I} \wedge e^{J} \wedge e^{K} \wedge e^{L} \epsilon_{I J K L}}_{\text {Cosmological constant }},
\end{aligned}
$$

where $\alpha_{1}, \alpha_{2}$ and $\alpha_{5}$ have $M^{2}$ dimension, $\alpha_{3}$ and $\alpha_{4}$ are real dimensionless parameters, and $\alpha_{6}$ is proportional to the cosmological constant. It is a remarkable feature that only finitely many terms are allowed by the symmetry once first order variables are chosen. This is in clear contrast with the formulation of pure-gravity in terms of metric variables where the most general action has infinitely many (higher-curvature) contributions ${ }^{1}$. We should point out that special case of the previous general action have been studied in the literature by Montesinos [9] and more recently by Date et al. in [10] and by Mercuri in [11] (the last two references consider coupling with fermion). We will discuss in detail these special cases a the end of this paper.

Notice that the terms proportional to $\alpha_{3}, \alpha_{4}$, and $\alpha_{5}$ are the Pontrjagin, the Euler, and the Nieh-Yan classes respectively. As the term proportional to $\alpha_{2}$, these topological invariants have no effect on the equations of motion of gravity as they can be written as the exterior derivative of suitable 3-forms (see equation (3) below). However, these boundary terms affect the canonical structure of the theory: they act as generating functionals of canonical transformations. As mentioned above this might have physical relevance when these canonical transformations cannot be unitarily implemented in the quantum theory. Heuristically, the off-shell contributions of the topological terms in (2) to transition amplitudes (in the language of the functional integral) might have non trivial effect in quantum gravity.

The paper is organized as follows: In the following section we perform the canonical analysis of the action (2). In addition to providing a complete analysis of the effect of the addition

\footnotetext{
${ }^{1}$ Incidentally, this would imply the renormalizability of quantum gravity if in the construction of the quantum theory one could find a regularization prescription compatible with the symmetries of [2].
} 
of topological invariants to the Holst action (completing existing existing results in the literature 9, 10] ), this section provides a detailed presentation of Holst's results [5] in a way that is alternative to the formulation of Barros e Sa [6]. As there are second class constraints that, for general values of the couplings, cannot be explicitly solved in Section III we compute the Dirac brackets in all generality. In Section IV we specialize to the family of couplings for which second class constraints can be solved, and we show that the term leading to the $\theta$ parameter in gravity cannot be obtained for real couplings. We discuss the way in which the $\theta$ term can be introduced at the Lagrangian level in Section D. We conclude with a discussion of our results in Section VI

\section{CANONICAL ANALYSIS}

The first step in trying to understand the effects of the topological terms added to the Holst action is to perform the canonical analysis of our action. In order to do this it will be convenient to write the topological terms in (2) explicitly as exterior derivatives of 3-forms, namely

$$
\begin{aligned}
S[e, \omega] & =\int \overbrace{\alpha_{1} \star\left(e^{I} \wedge e^{J}\right) \wedge F_{J I}(\omega)+\alpha_{2} e^{I} \wedge e^{J} \wedge F_{I J}(\omega)}^{\text {Holst }} \\
& +\left(\alpha_{3}-i \alpha_{4}\right) d \mathscr{L}_{C S}\left(\omega^{A S D}\right)+\left(\alpha_{3}+i \alpha_{4}\right) d \mathscr{L}_{C S}\left(\omega^{S D}\right)+\alpha_{5} d\left(d_{\omega} e^{I} \wedge e_{I}\right),
\end{aligned}
$$

where $\omega^{S D}=i(\star \omega)^{S D}=\omega-i(\star \omega)$ and $\omega^{A S D}=i(\star \omega)^{A S D}=\omega+i(\star \omega)$ are the self-dual and anti-self dual parts of the Lorentz connection $\omega$ respectively, and

$$
\mathscr{L}_{C S}(\omega)=\omega_{I J} \wedge d \omega^{I J}+\frac{2}{3} \omega_{I J} \wedge[\omega \wedge \omega]^{I J}
$$

is the Chern-Simons Lagrangian density. Despite of the presence of complex variables in the above expression of the action, the action principle is manifestly real as the strict equality with (2) holds. In performing the canonical analysis of our theory we will use both (2) as well as (3) according to convenience.

As mentioned above, the addition of boundary terms to the action principle induces canonical transformations in the phase space formulation. Notice that the terms added to Holst's formulation are the most general total differentials that one can write using the fields $e$ and $\omega$ without breaking local Lorentz invariance and diffeomorphism invariance. Therefore, studying the canonical structure behind (2) amounts for studying the most general set of possible canonical transformations compatible with the field content and symmetries of the action.

We assume that the spacetime manifold has topology $\mathscr{M}=\Sigma \times \mathbb{R}$, with $\Sigma$ compact. In order to perform the Hamiltonian formulation we start by doing the customary $(3+1)$ decomposition consisting of choosing an arbitrary foliation of spacetime in terms of the level hyper-surfaces of a global time function $t$. The hyper-surfaces $t=$ constant will be denoted $\Sigma$ as well. We denote $n^{a}$ the normal to the foliation. The arbitrariness in the choice of foliation is encoded in the lapse scalar $N$ and the shift vector (tangent to the foliation) $N^{a}$ which imply that the time vector $t^{a}$ (defined by $t^{a}(t)=1$ ) takes the form $t^{a}=N^{a}+N n^{a}$. This implies that the following equation for the projection of the tetrad in the $t^{a}$ direction:

$$
e_{t}^{I}=N n^{I}+e_{a}^{I} N^{a}
$$

where $n^{I} \equiv n^{a} e_{a}^{I}$. With these definitions the Holst action (for the moment we are ignoring the topological terms, i.e. taking $\alpha_{3}=\alpha_{4}=\alpha_{5}=0$ ) takes the simple form:

$$
\mathscr{L}_{H}=2 \epsilon^{a b c} e_{a}^{I} e_{b}^{J} q_{I J K L} F_{t c}^{K L}+2 N \epsilon^{a b c} n^{I} e_{a}^{J} q_{I J K L} F_{b c}^{K L}+2 N^{d} \epsilon^{a b c} e_{d}^{I} e_{a}^{J} q_{I J K L} F_{b c}^{K L},
$$


where $q_{I J K L}=\alpha_{1} \epsilon_{I J K L}+\alpha_{2} \eta_{I J K L}$ denoting by $\eta_{I J K L}=\eta_{[I|K|} \eta_{J] L}$ the invariant metric in the Lie algebra of the Lorentz group. If we define $\Pi_{K L}^{a}=2 \epsilon^{a b c} e_{b}^{I} e_{c}^{J} q_{I J K L}$ then the previous action takes the form

$$
\begin{aligned}
\mathscr{L}_{H}=\Pi_{I}^{a} \dot{e}_{a}^{I}+\Pi_{I J}^{a} \dot{\omega}_{a}^{I J}+N^{I J} D_{a} \Pi_{I J}^{a}+ & 2 N \epsilon^{a b c} n^{I} e_{a}^{J} q_{I J K L} F_{b c}^{K L}+N^{a} \Pi_{I J}^{b} F_{b a}^{I J} \\
& +{ }^{(1)} \lambda_{a}^{I} \Pi_{I}^{a}+{ }^{(2)} \lambda_{a}^{K L}\left(\Pi_{K L}^{a}-2 \epsilon^{a b c} e_{b}^{I} e_{c}^{J} q_{I J K L}\right),
\end{aligned}
$$

where $N^{I J} \equiv \omega_{t}^{I J}, N, N^{a}$, ${ }^{(1)} \lambda_{a}^{I}$, and ${ }^{(2)} \lambda_{a}^{K L}$ are Lagrange multipliers imposing the primary constraints of the Holst action. On the other hand $\Pi_{I J}^{a}$ and $\Pi_{I}^{a}$ denote the momentum conjugate to $\omega_{a}^{I J}$ and $e_{a}^{I}$ respectively. Therefore, the primary constraints are

$$
\begin{aligned}
& \Pi_{I}^{a} \approx 0 \\
& \Pi_{K L}^{a}-2 \epsilon^{a b c} e_{b}^{I} e_{c}^{J} q_{I J K L} \approx 0 \\
& \text { Lorentz-Gauss law } \quad D_{a} \Pi_{I J}^{a}=2 D_{a}\left(\epsilon^{a b c} e_{b}^{I} e_{c}^{J} q_{I J K L}\right) \approx 0 \\
& \text { Vector constraint } \quad \epsilon^{a b c} e_{d}^{I} e_{a}^{J} q_{I J K L} F_{b c}^{K L} \approx 0 \\
& \text { Scalar constraint } \quad \epsilon^{a b c} n^{I} e_{a}^{J} q_{I J K L} F_{b c}^{K L} \approx 0 .
\end{aligned}
$$

A simple look at the list of primary constraints tell us that there will be secondary constraints when we require the primary to be preserved by the Hamiltonian evolution. However, before continuing and completing the analysis it will be convenient to treat the general case including the topological terms.

\section{A. The Holst action plus topological terms}

Including the topological terms is straightforward at this level. According to (3), and using that $\partial \Sigma=0$, the Lagrangian (5) is modified by the addition of the total time derivative, namely

$$
\mathscr{L}=\mathscr{L}_{H}+\left(\alpha_{3}-i \alpha_{4}\right) \partial_{t} \mathscr{L}_{C S}\left(\omega^{A S D}\right)+\left(\alpha_{3}+i \alpha_{4}\right) \partial_{t} \mathscr{L}_{C S}\left(\omega^{S D}\right)+\alpha_{5} \partial_{t}\left(d_{\omega} e^{I} \wedge e_{I}\right)
$$

which, using that $\partial_{t} \mathscr{L}_{C S}(\omega)=2 B_{I J}^{a}(\omega) \dot{\omega}_{a}^{I J}$ where $B_{I J}^{a}=\epsilon^{a b c} F_{b c}(\omega)$, implies that the conjugate momenta $\Pi_{I J}^{a}$ and $\Pi_{I}^{a}$ receive additional contributions of the form $4 \alpha_{3} B_{I J}^{a}+4 \alpha_{4} \epsilon_{I J}^{K L} B_{K L}^{a}+$ $\alpha_{5} \epsilon^{a b c}\left(e_{b}\right)_{[I}\left(e_{b}\right)_{J]}$ and $2 \alpha_{5} \epsilon^{a b c} d_{\omega} e_{b c}^{I}$ respectively. Notice that, due to $\partial \Sigma=0$, the addition of the topological terms only affects the kinetic term of the Holst action. More precisely if we define the real functional

$$
W\left(\omega_{a}^{I J}, e_{a}^{I}\right)=\int_{\Sigma}\left(\alpha_{3}-i \alpha_{4}\right) \mathscr{L}_{C S}\left(\omega^{A S D}\right)+\left(\alpha_{3}+i \alpha_{4}\right) \mathscr{L}_{C S}\left(\omega^{S D}\right)+\alpha_{5}\left(d_{\omega} e^{I} \wedge e_{I}\right),
$$

the new constraints become

$$
\begin{aligned}
& \Pi_{I}^{a}-\frac{\delta W}{\delta e_{a}^{I}}= \\
& =\Pi_{I}^{a}-2 \alpha_{5} \epsilon^{a b c}\left(d_{\omega} e\right)_{b c I} \approx 0 \\
& \Pi_{I J}^{a}-2 \epsilon^{a b c} e_{b}^{K} e_{c}^{L} q_{I J K L}-\frac{\delta W}{\delta \omega_{a}^{I J}}= \\
& =\Pi_{K L}^{a}-2 \epsilon^{a b c} e_{b}^{I} e_{c}^{J} q_{I J K L}-\alpha_{5} \epsilon^{a b c}\left(e_{b}\right)_{[K}\left(e_{b}\right)_{L]}-4 \alpha_{3} B_{K L}^{a}-4 \alpha_{4} \epsilon_{K L}^{I J} B_{I J}^{a} \approx 0 \\
& \text { Lorentz-Gauss law } \quad D_{a} \Pi_{I J}^{a}=2 D_{a}\left(\epsilon^{a b c} e_{b}^{I} e_{c}^{J} q_{I J K L}\right) \approx 0 \\
& \text { Vector constraint } \epsilon^{a b c} e_{d}^{I} e_{a}^{J} q_{I J K L} F_{b c}^{K L} \approx 0 \\
& \text { Scalar constraint } \epsilon^{a b c} n^{I} e_{a}^{J} q_{I J K L} F_{b c}^{K L} \approx 0 .
\end{aligned}
$$


Notice that the addition of the topological invariants, being just boundary terms, modify only the constraints defining the momenta. The vector and scalar constraints remain the same as they do not depend on momentum variables at this stage ( $e_{a}^{I}$ is here considered a configuration variable). The Gauss law (16) does depend on the momenta (as written as in the 1.h.s.); however, it also remains unchanged (as in the r.h.s.) due to the Bianchi identity implying $D_{a} B_{I J}^{a}=0$.

\section{B. The time gauge: reducing $S O(3,1)$ to $S O(3)$}

Let us now introduce the standard gauge condition that reduces the Lorentz gauge symmetry to an $S O(3)$ gauge symmetry. The gauge condition is often called the time-gauge condition. It corresponds to the requirement that the zeroth element of the tetrad coincide with the co-normal to $\Sigma$, namely $n_{I} e_{\mu}^{I}=n_{\mu}$. This implies the phase-space additional gauge-fixing constraint

$$
n_{I} e_{a}^{I} \approx 0
$$

which now must be added to the list of primary constraint above. The previous gauge fixing condition is necessary to recover the compact gauge group connection variables that are used in LQG. One can of course complete the Hamiltonian formulation without breaking the local Lorentz invariance. However, the price to be paid is a non trivial Dirac bracket between the components the Lorentz connection $\omega_{a}^{I J}$ [7] precluding the existence of a connection representation in the quantum theory. A proposal for quantizing the non-commutative connection can be found in [8].

The condition (19) is second class with respect to the projection of equation (7) in the $n^{I}$ internal direction. In other words the requirement that the gauge (19) is preserved in time fixes the Lagrange multipliers ${ }^{(1)} \lambda_{I}^{a}$ in (6). This means that we can impose $n^{I} \Pi_{I}^{a}=0$ and $n_{I} e_{a}^{I}=0$ strongly. From now on we will take $n^{I}=(1,0,0,0)$ and denote with lower case Latin alphabet letters the space-like internal directions. The new restricted dynamical system is described by

$$
\begin{aligned}
& \Pi_{i}^{a}-2 \alpha_{5} \epsilon^{a b c}\left(d_{\omega} e\right)_{b c i} \approx 0 \\
& \frac{1}{2} \epsilon_{k}^{i j} \Pi_{i j}^{a} \approx\left(2 \alpha_{2}-\alpha_{5}\right) \epsilon^{a b c} e_{b}^{i} e_{c}^{j} \epsilon_{i j k}+4 \alpha_{3} \epsilon_{k}^{i j} B_{i j}^{a}-16 \alpha_{4} B_{k 0}^{a} \\
& \Pi_{k 0}^{a} \approx 2 \alpha_{1} \epsilon^{a b c} e_{b}^{i} e_{c}^{j} \epsilon_{i j k}+4 \alpha_{3} B_{k 0}^{a}-4 \alpha_{4} \epsilon_{k}{ }^{l m} B_{l m}^{a} \\
& \text { Lorentz-Gauss law }\left\{\begin{array}{c}
\epsilon_{m l k} E^{a l} \hat{K}_{a}^{k} \approx 0, \\
\partial_{d} E^{d k}+\epsilon_{i j k} \hat{\Gamma}_{d}^{i} E^{d j} \approx 0,
\end{array}\right. \\
& \text { Vector constraint } \Pi_{i j}^{b} F_{b a}^{i j}+2 \Pi_{i 0}^{b} F_{b a}^{i 0} \approx 0 \\
& \text { Scalar constraint } \epsilon^{a b c} e_{a}^{i} q_{0 i j k} F_{b c}^{j k}+2 \epsilon^{a b c} e_{a}^{i} q_{0 i 0 k} F_{b c}^{0 k} \approx 0,
\end{aligned}
$$

where we have used the following definitions

$$
E_{i}^{a} \equiv \frac{1}{2} \epsilon^{a b c} e_{a}^{j} e_{b}^{k} \epsilon_{i j k}, \quad \hat{K}_{a}^{i} \equiv \omega_{a}^{0 i} \quad \hat{\Gamma}_{a}^{i} \equiv \frac{1}{2} \epsilon_{j k}^{i} \omega_{a}^{j k}
$$

and the Bianchi identity to write the Gauss law constraints (23). We will see in a moment that the previous variables are indeed the extrinsic curvature component and the Levi-Civita spin connection respectively, which justifies the notation. Equations (21) and (22) can be combined in a way to simplify the dependence on the triad $e_{a}^{i}$ : notice that the triad dependence is the same in both equations. Therefore, one can introduce new variables

$$
\begin{aligned}
{ }^{ \pm \mathscr{P}_{i}^{a}} & \equiv \frac{1}{4} \epsilon_{i}{ }^{j k} \Pi_{j k}^{a} \pm \frac{2 \alpha_{2}+\alpha_{5}}{4 \alpha_{1}} \Pi_{i 0}^{a} \\
& =\frac{1}{4} \epsilon_{i}{ }^{j k} \Pi_{j k}^{a} \pm \frac{1}{2 \gamma} \Pi_{i 0}^{a},
\end{aligned}
$$


where we introduced the definition $\gamma \equiv \frac{2 \alpha_{1}}{2 \alpha_{2}-\alpha_{5}}$. The previous new momenta are the conjugate of new $S O(3)$ connections

$$
{ }^{ \pm} \omega_{d l}= \pm \gamma \omega_{d l 0}+\frac{1}{2} \epsilon_{l}{ }^{m n} \omega_{d m n},
$$

and we recognize $\gamma$ as the Immirzi parameter at this stage. In the time gauge, one can write the functional $W\left(\omega_{a}^{I J}, e_{a}^{I}\right)$ defined in (13) as a functional as $W\left(\omega_{a}^{I J}, e_{a}^{I}\right)=W_{0}\left({ }^{+} \omega_{a}{ }^{i},{ }^{-} \omega_{a}^{i}\right)+$ $\alpha_{5} \epsilon^{a b c}\left(d_{\omega} e\right)_{a b}^{i} e_{c i}$ where $W_{0}\left({ }^{+} \omega_{a}^{i},{ }^{-} \omega_{a}^{i}\right)$ is simply the value of $W\left(\omega_{a}^{I J}, e_{a}^{I}\right)$ for $\alpha_{5}=0$. Using the new variables the constraints become

$$
\begin{aligned}
& (\mathbb{I})_{i}^{a} \equiv \Pi_{i}^{a}-2 \alpha_{5} \epsilon^{a b c}\left(d_{\omega} e\right)_{b c i} \approx 0 \\
& (\mathbb{I I I})_{k}^{a} \equiv \mathscr{P}_{k}^{a}-2 \frac{\alpha_{1}}{\gamma} \epsilon^{a b c} e_{b}^{i} e_{c}^{j} \epsilon_{i j k}-\frac{\delta W_{0}}{\delta+\omega_{a}^{i}} \\
& (\mathbb{I I I I})_{k}^{a} \equiv-\mathscr{P}_{k}^{a}-\frac{\delta W_{0}}{\delta-\omega_{a}^{i}} \\
& \text { Boosts constraint } \quad B^{k} \equiv \partial_{d} E^{d k}-\epsilon_{i j k} \hat{\Gamma}_{d}^{i} E^{d j} \approx 0 \Longrightarrow B^{k}=-\epsilon_{i j k} E^{a i}\left(\hat{\Gamma}_{a}^{j}-\Gamma_{a}^{j}\right) \approx 0 \\
& S O(3) \text { Gauss law } \quad G_{m} \equiv \epsilon_{m l k} E^{a l} \hat{K}_{a}^{k} \approx 0 \\
& \text { Vector constraint } \quad V_{a} \equiv \Pi_{i j}^{b} F_{b a}^{i j}+2 \Pi_{i 0}^{b} F_{b a}^{i 0} \approx 0 \\
& \text { Scalar constraint } \quad S \equiv \epsilon^{a b c} e_{a}^{i} q_{0 i j k} F_{b c}^{j k}+2 \epsilon^{a b c} e_{a}^{i} q_{0 i 0 k} F_{b c}^{0 k} \approx 0,
\end{aligned}
$$

where

$$
\hat{K}_{a}^{i}=\frac{1}{2 \gamma}\left({ }^{+} \omega_{a}^{i}-{ }^{-} \omega_{a}^{i}\right) \quad \hat{\Gamma}_{a}^{i}=\frac{1}{2}\left({ }^{-} \omega_{a}^{i}+{ }^{+} \omega_{a}^{i}\right) .
$$

Notice that we have re-written the boost part of the Lorentzian Gauss law - which we should expect to be second class due to the time gauge condition (19) - in terms of the spin connection $\Gamma_{a}^{i}$, i.e., the solution of Cartan's first structure equation

$$
\partial_{[a} e_{b]}^{k}-\epsilon^{k}{ }_{i j} \Gamma_{[a}^{i} e_{b]}^{j}=0 .
$$

Indeed it will be convenient to introduce the quantity

$$
(\mathbb{I V})_{a}^{i} \equiv \hat{\Gamma}_{a}^{i}-\Gamma_{a}^{i} .
$$

We will explicitly show in what follows how three components of the boost part of the Lorentzian Gauss law plus six secondary constraints (not derived yet) imply $(\mathbb{I} \mathbb{V})_{a}^{i} \approx 0$ which will be shown to be second class.

By setting $W=0$ one recovers the primary constraints of Holst $[5]$. Notice in addition that, as mentioned above, only the first three constraints in the previous list are modified by the addition of the Pontrjagin, Euler and Nieh-Yan invariants to the Holst action. The modification is very simple: if we take $\left\{{ }^{+} \omega_{a}^{i},{ }^{-} \omega_{a}^{i}, e_{a}^{i}\right\}$ as configuration variables then, the Holst momenta are shifted according to $p \rightarrow p+\left\{p, W\left({ }^{+} \omega_{a}^{i},{ }^{-} \omega_{a}^{k}, e_{a}^{i}\right)\right\}$, where $p$ denotes ${ }^{+} \mathscr{P}_{i}^{a},-\mathscr{P}_{i}^{a}$, and $\Pi_{i}^{a}$. The modification introduced by the topological invariants is just a canonical transformation generated by $W\left({ }^{+} \omega_{a}^{i},-\omega_{a}^{k}, e_{a}^{i}\right)$. For that reason, the constraint algebra is not affected by the topological terms. Therefore, the consistency conditions (secondary constraints) that follow from requiring that primary constraints are preserved by the total Hamiltonian remain unchanged. This also hold for the classification between first class and second class constraints. Collecting all primary constraints the Hamiltonian becomes

$$
H=\int_{\Sigma} N S+N^{a} V_{a}+N^{k} G_{k}+\lambda^{k} B_{k}+\lambda_{d l}^{(1)}(\mathbb{I})^{d l}+\lambda_{d l}^{(2)}(\mathbb{I I I})^{d l}+\lambda_{d l}^{(3)}(\mathbb{I I I I})^{d l},
$$


At this point one needs to look for potential secondary constraint by requiring that the constraint surface be preserved by the time evolution defined by the previous Hamiltonian. This leads to the following consistency conditions:

$$
\begin{aligned}
& 0 \approx\left\{(\mathbb{I})_{i}^{a}, H_{T}\right\}=\frac{4 \alpha_{1}}{\gamma} \epsilon^{a b c} \lambda_{b}^{(2) j} e_{c}^{k} \epsilon_{i j k}+\text { additional terms } \\
& 0 \approx\left\{(\mathbb{I I I})_{i}^{a}, H_{T}\right\}=\frac{1}{2} \lambda^{j} E^{a k} \epsilon_{i j k}-\left(\alpha_{5}+2\left(2 \alpha_{2}-\alpha_{5}\right)\right) \epsilon_{i j k} \epsilon^{a b c} e_{b}^{j} \lambda_{c}^{(1) k}-\frac{\delta H_{0}}{\delta^{+} \omega_{a}^{k}} \\
& 0 \approx\left\{(\mathbb{I I I I})_{i}^{a}, H_{T}\right\}=\frac{1}{2} \lambda^{j} E^{a k} \epsilon_{i j k}-\alpha_{5} \epsilon_{i j k} \epsilon^{a b c} e_{b}^{j} \lambda_{c}^{(1) k}-\frac{\delta H_{0}}{\delta-\omega_{a}^{k}} \\
& 0 \approx\left\{B_{i}, H_{T}\right\}=-\frac{1}{2} \epsilon_{i j k} \lambda_{b}^{(3) j} E^{b k}+\text { additional terms, }
\end{aligned}
$$

where $H_{0} \equiv \int_{\Sigma} N S+N^{a} V_{a}+N^{k} G_{k}$ is a linear combination of the scalar, vector and $S O(3)$ Gauss constraints. Equations (40) and (41) completely determine the Lagrange multipliers $\lambda_{b}^{(2) j}$ and $\lambda_{b}^{(1) j}$ respectively. Equation (43) determines the antisymmetric part of $\lambda_{i j}^{(3)} \equiv \lambda_{b i}^{(2)} E_{j}^{b}$, i.e., it fixes three out of the nine components of the Lagrange multiplier $\lambda_{b}^{(3) j}$. Hence, there are no secondary constraints arising from these equations.

Equation (42) leads to secondary constraints. To see this one has to replace in (42) the solution for $\lambda_{a}^{(1) i}$ obtained from (41). Consider the quantity

$$
C_{i j} \equiv\left(e_{a}\right)_{j}\left\{(\mathbb{I I I I})_{i}^{a}, H_{T}\right\}
$$

Then it is easy to see that the three conditions $C_{[i j]}=0$ can be used to fix the Lagrange multipliers $\lambda^{i}$ while the remaining six conditions $C_{(i j)}=0$ are proportional to

$$
C_{(i j)}=\left(e_{a}\right)_{(j}\left\{(\mathbb{I I I I})_{i)}^{a}, H_{T}\right\} \propto \epsilon^{a b d} E_{d(m} d_{a}^{(\hat{\Gamma})} e_{b l)} \approx 0
$$

where $d^{\hat{\Gamma}}$ is the exterior covariant differential computed with $\hat{\Gamma}$. Notice now that the six independent above constraints over $\hat{\Gamma}$ can be combined with the three boost constraints $B^{i}$ in (32) into the nine component constraint (38), namely

$$
B^{i}=0 \quad \text { in addition to } \quad C_{(i j)}=0 \quad \Leftrightarrow \quad(\mathbb{I} \mathbb{V})_{a}^{i} \equiv \hat{\Gamma}_{a}^{i}-\Gamma_{a}^{i}=0 .
$$

We can therefore rearrange the total Hamiltonian in the more convenient form

$$
H_{T}=\int_{\Sigma} N S+N^{a} V_{a}+N^{k} G_{k}+\lambda_{d l}^{(1)}(\mathbb{I})^{d l}+\lambda_{d l}^{(2)}(\mathbb{I I})^{d l}+\lambda_{d l}^{(3)}(\mathbb{I} \mathbb{I I})^{d l}+\lambda_{l}^{(4) d}(\mathbb{I} \mathbb{V})_{d}^{l}
$$

where instead of adding the secondary constraint (45) to the primary constraints Hamiltonian, we have dropped the term $\lambda^{k} B_{k}$ from the integrand in (39) and added the term $\lambda_{l}^{(4) d}(\mathbb{I} \mathbb{V})_{d}^{l}$ in the previous expression of the total Hamiltonian. One can check that the consistency conditions of the new set of constraints fix the Lagrange multipliers $\lambda^{(\mu)}$ for $\mu=1,2,3,4$, while the Lagrange multipliers $N, N^{a}$ and $N^{k}$ are left arbitrary. From this one concludes that the 36 constraints $(\mathbb{I})_{i}^{a}$, $(\mathbb{I I I})_{i}^{a},(\mathbb{I I I})_{i}^{a}$, and $(\mathbb{I} \mathbb{V})_{a}^{i}$ are second class constraint while the seven remaining constraints (the scalar $S$, vector $V_{a}$ and Gauss $G_{k}$ constraints) are first class ${ }^{2}$. There are 27 configuration variables $e_{a}^{i},{ }^{+} \omega_{a}^{i}$, and $-\omega_{a}^{i}$; therefore, the counting of degrees of freedom yields the expected two degrees of freedom of gravity.

\footnotetext{
2 Strictly speaking the scalar $S$, vector $V_{a}$ and Gauss $G_{k}$ constraints are not first class as written here. In order to make them into first class constraints one would need to add to them appropriate linear combinations of the
} 


\section{CONSTRUCTION OF THE DIRAC BRACKET}

The analysis up to this point follows the same logical line as in the Holst's case, by the simple fact that the addition of a surface term to the action does not change the Poisson brackets among the constraints. However, contrary to the Holst case, we cannot explicitly solve the constraint $(\mathbb{I I I})_{i}^{a}$. The reason is the presence of the curvature tensor in the magnetic field contributions to (30) which prevents one from eliminating the densitized triad as a function of the connection and its momenta: the dependence on the densitized triad on is quite complicated due to the non polynomial character of the spin connection $\Gamma_{a}^{i}(E)$. Thus, in order to complete the canonical analysis of the general action, we need to explicitly construct the Dirac brackets for the second class constraints $(\mathbb{I})_{i}^{a},(\mathbb{I I I})_{i}^{a},(\mathbb{I I I I})_{i}^{a}$, and $(\mathbb{I} \mathbb{V})_{a}^{i}$. Before computing the constraint algebra it will be convenient to replace the constraint $(\mathbb{I})_{i}^{a}$. The constraint algebra is

$$
\begin{aligned}
& \left\{(\mathbb{I})_{i}^{a},(\mathbb{I})_{j}^{b}\right\}=0, \\
& \left\{(\mathbb{I})_{i}^{a},(\mathbb{I} \mathbb{I})_{j}^{b}\right\}=\left(4 \alpha_{2}-\alpha_{5}\right) \epsilon^{a b c} \epsilon_{i j k} e_{c}^{k} \delta^{3}(x, y), \\
& \left\{(\mathbb{I})_{i}^{a},(\mathbb{I} \mathbb{I} \mathbb{I})_{j}^{b}\right\}=\alpha_{5} \epsilon^{a b c} \epsilon_{i j k} e_{c}^{k} \delta^{3}(x, y), \\
& \left\{(\mathbb{I})_{i}^{a},(\mathbb{I} \mathbb{V})_{b}^{j}\right\}=\frac{\delta \Gamma_{b}^{j}(y)}{\delta e_{a}^{i}(x)}, \\
& \left\{(\mathbb{I I})_{i}^{a},(\mathbb{I} \mathbb{I})_{j}^{b}\right\}=0, \\
& \left\{(\mathbb{I I})_{i}^{a},(\mathbb{I} \mathbb{I})_{j}^{b}\right\}=0, \\
& \left\{(\mathbb{I I I})_{i}^{a},(\mathbb{I} \mathbb{V})_{b}^{j}\right\}=-\frac{1}{2} \delta_{a}^{b} \delta_{i}^{j} \delta^{3}(x, y), \\
& \left\{(\mathbb{I I I I})_{i}^{a},(\mathbb{I} \mathbb{I} \mathbb{I})_{j}^{b}\right\}=0, \\
& \left\{(\mathbb{I I I I})_{i}^{a},(\mathbb{I} \mathbb{V})_{b}^{j}\right\}=-\frac{1}{2} \delta_{a}^{b} \delta_{i}^{j} \delta^{3}(x, y), \\
& \left\{(\mathbb{I} \mathbb{V})_{i}^{a},(\mathbb{I} \mathbb{V})_{j}^{b}\right\}=0 .
\end{aligned}
$$

second class constraints. Nevertheless, the fact that $N, N^{a}$ and $N^{k}$ are not fixed by the equations of motion implies the existence of 7 first class constraints and that these coincide with the scalar $S$, vector $V_{a}$ and Gauss $G_{k}$ constraints once the second class constraints have been solved. To see this in a more general way, suppose we have two sets of constraints, $\phi_{A}$ and $\theta_{A}$ such that

$$
\begin{aligned}
\left\{\phi_{A}, \phi_{B}\right\} & =0, \\
\left\{\phi_{A}, \theta_{B}\right\} & =M_{A B}, \\
\left\{\theta_{A}, \theta_{B}\right\} & =\Delta_{A B},
\end{aligned}
$$

where $\Delta$ is an invertible matrix and $M$ not. We can 'decouple' the two sets of constraints by the redefinition:

$$
\phi_{A} \rightarrow \tilde{\phi}_{A}=\phi_{A}-M_{A C}\left(\Delta^{-1}\right)^{C D} \theta_{D}
$$

the algebra becomes:

$$
\begin{aligned}
\left\{\tilde{\phi}_{A}, \tilde{\phi}_{B}\right\} & =\left\{\phi_{A}-M_{A C}\left(\Delta^{-1}\right)^{C D} \theta_{D}, \phi_{B}-M_{B C}\left(\Delta^{-1}\right)^{C D} \theta_{D}\right\} \approx 0, \\
\left\{\tilde{\phi}_{A}, \theta_{B}\right\} & =-\left\{M_{A C}\left(\Delta^{-1}\right)^{C D}, \theta_{B}\right\} \theta_{D} \approx 0, \\
\left\{\theta_{A}, \theta_{B}\right\} & =\Delta_{A B},
\end{aligned}
$$

this is completely equivalent to solve first the Dirac brackets for the $\theta_{A}$ sector and then, recomputing the remaining algebra for the $\phi_{A}$ sector. 
We construct the Dirac matrix, which we represent symbolically as

$$
\mathbf{M}=\left(\begin{array}{cccc}
0 & \left(4 \alpha_{2}-\alpha_{5}\right) A_{i j}^{a b}(x, y) & \alpha_{5} A_{i j}^{a b}(x, y) & B_{a l}^{b k}(x, y) \\
-\left(4 \alpha_{2}-\alpha_{5}\right) A_{i j}^{a b}(x, y) & 0 & 0 & -\frac{1}{2} I_{a l}^{b k}(x, y) \\
-\alpha_{5} A_{i j}^{a b}(x, y) & 0 & 0 & -\frac{1}{2} I_{a l}^{b k}(x, y) \\
-B_{a l}^{b k}(x, y) & \frac{1}{2} I_{a l}^{b k}(x, y) & \frac{1}{2} I_{a l}^{b k}(x, y) & 0
\end{array}\right)
$$

where

$$
\begin{gathered}
I_{a l}^{b k}(x, y)=\delta_{a}^{b} \delta_{l}^{k} \delta^{3}(x, y), \\
A_{i j}^{a b}(x, y)=\epsilon^{a b c} \epsilon_{i j k} e_{c}^{k} \delta^{3}(x, y),
\end{gathered}
$$

and

$$
B_{a l}^{b k}(x, y) \equiv \frac{\delta \Gamma_{a}^{k}(x)}{\delta e_{b}^{l}(y)}
$$

The inverse of this matrix is:

$\mathbb{M}^{-1}=\frac{\gamma}{4 \alpha_{1}}\left(\begin{array}{cccc}0 & -\left(A^{-1}\right)_{a b}^{i j}(x, y) & \left(A^{-1}\right)_{a b}^{i j}(x, y) & 0 \\ \left(A^{-1}\right)_{a b}^{i j}(x, y) & 0 & -2\left(A^{-1} \cdot B\right)_{a b}^{k l}(x, y) & -2 \alpha_{5} I_{a l}^{b k}(x, y) \\ -\left(A^{-1}\right)_{a b}^{i j}(x, y) & 2\left(A^{-1} \cdot B\right)_{a b}^{k l}(x, y) & 0 & 2\left(4 \alpha_{2}-\alpha_{5}\right) I_{a l}^{b k}(x, y) \\ 0 & 2 \alpha_{5} I_{a l}^{b k}(x, y) & -2\left(4 \alpha_{2}-\alpha_{5}\right) I_{a l}^{b k}(x, y) & 0\end{array}\right)$,

where the dot in the above expression involves the appropriate index contraction and integration over $\Sigma$, explicitly:

$$
\left(A^{-1} \cdot B\right)_{a b}^{k l}(x, y) \equiv \int d z\left(A^{-1}\right)_{a c}^{k m}(x, z) B_{b m}^{c l}(z, y)
$$

The only explicit inversion that one needs is that of the tensor density $A_{i j}^{a b}(x, y)$. It is straightforward to show that the inverse is given by

$$
\left(A^{-1}\right)_{a b}^{i j}(x, y)=\frac{\delta e_{a}^{i}(x)}{\delta E_{j}^{b}(y)},
$$

which can be computed explicitly using that $e_{a}^{i}=\frac{1}{2} \epsilon_{a b c} \epsilon^{i j k} E_{j}^{b} E_{k}^{c} /(\sqrt{\operatorname{det}(E)})$ as implied by eq. (26). Notice also that the previous equation implies

$$
\left(A^{-1} \cdot B\right)_{a b}^{k l}(x, y)=\frac{\delta \Gamma_{a}^{k}(x)}{\delta E_{l}^{b}(y)} .
$$

Thus, the full Dirac bracket is given by

$$
\begin{aligned}
\{f, g\}_{D} & =\{f, g\}-\frac{\gamma}{4 \alpha_{1}} \int\left[-\left\{f,(\mathbb{I})_{i}^{a}(x)\right\} \frac{\delta e_{a}^{i}(x)}{\delta E_{j}^{b}(y)}\left\{(\mathbb{I I})_{j}^{b}(y), g\right\}+\left\{f,(\mathbb{I})_{i}^{a}(x)\right\} \frac{\delta e_{a}^{i}(x)}{\delta E_{j}^{b}(y)}\left\{(\mathbb{I} \mathbb{I})_{j}^{b}(y), g\right\}\right. \\
& +\left\{f,(\mathbb{I I})_{i}^{a}(x)\right\} \frac{\delta e_{a}^{i}(x)}{\delta E_{j}^{b}(y)}\left\{(\mathbb{I})_{j}^{b}(y), g\right\}-2\left\{f,(\mathbb{I} \mathbb{I})_{i}^{a}(x)\right\} \frac{\delta \Gamma_{a}^{i}(x)}{\delta E_{j}^{b}(y)}\left\{(\mathbb{I} \mathbb{I})_{j}^{b}(y), g\right\} \\
& -2 \alpha_{5}\left\{f,(\mathbb{I} \mathbb{I})_{i}^{a}(x)\right\}\left\{(\mathbb{I} \mathbb{V})_{a}^{i}(y), g\right\}-\left\{f,(\mathbb{I} \mathbb{I} \mathbb{I})_{i}^{a}(x)\right\} \frac{\delta e_{a}^{i}(x)}{\delta E_{j}^{b}(y)}\left\{(\mathbb{I})_{j}^{b}(y), g\right\} \\
& +2\left\{f,(\mathbb{I} \mathbb{I} \mathbb{I})_{i}^{a}(x)\right\} \frac{\delta \Gamma_{a}^{i}(x)}{\delta E_{j}^{b}(y)}\left\{(\mathbb{I})_{j}^{b}(y), g\right\}+2\left(4 \alpha_{2}-\alpha_{5}\right)\left\{f,(\mathbb{I} \mathbb{I})_{i}^{a}(x)\right\}\left\{(\mathbb{I} \mathbb{V})_{a}^{i}(y), g\right\} \\
& \left.+2 \alpha_{5}\left\{f,(\mathbb{I} \mathbb{V})_{i}^{a}(x)\right\}\left\{(\mathbb{I})_{i}^{a}(y), g\right\}-2\left(4 \alpha_{2}-\alpha_{5}\right)\left\{f,(\mathbb{I} \mathbb{V})_{i}^{a}(x)\right\}\left\{(\mathbb{I} \mathbb{I})_{a}^{i}(y), g\right\} \cdot\right] d^{3} x d^{3} y .(61)
\end{aligned}
$$


From (61) we obtain the new commutation relations:

$$
\begin{aligned}
& \left\{{ }^{+} \omega_{a}^{i}(x), e_{b}^{j}(y)\right\}_{D}=\frac{\gamma}{4 \alpha_{1}} \frac{\delta e_{a}^{i}(x)}{\delta E_{j}^{b}(y)}, \\
& \left\{{ }^{+} \omega_{a}^{i}(x),{ }^{+} \omega_{b}^{j}(y)\right\}_{D}=0, \\
& \left\{{ }^{+} \omega_{a}^{i}(x),{ }^{-} \omega_{b}^{j}(y)\right\}_{D}=\frac{\gamma}{2 \alpha_{1}} \frac{\delta \Gamma_{b}^{j}(y)}{\delta E_{k}^{a}(x)}, \\
& \left\{e_{a}^{i}(x), e_{b}^{j}(y)\right\}_{D}=0, \\
& \left\{e_{a}^{k}(x), \mathscr{P}_{j}^{b}(y)\right\}_{D}=-\frac{\gamma}{4 \alpha_{1}} \int \frac{\delta e_{d}^{m}(z)}{\delta E_{i}^{a}(x)} \frac{\delta}{\delta+\omega_{d}^{m}(z)}\left[\frac{\delta W_{0}}{\delta+\omega_{b}^{j}(y)}-\frac{\delta W_{0}}{\delta-\omega_{b}^{j}(y)}\right] d z, \\
& \left\{e_{a}^{k}(x),-\mathscr{P}_{j}^{b}(y)\right\}_{D}=-\frac{\gamma}{4 \alpha_{1}} \int \frac{\delta e_{d}^{m}(z)}{\delta E_{i}^{a}(x)} \frac{\delta}{\delta-\omega_{d}^{m}(z)}\left[\frac{\delta W_{0}}{\delta+\omega_{b}^{j}(y)}-\frac{\delta W_{0}}{\delta-\omega_{b}^{j}(y)}\right] d z, \\
& \left\{+\mathscr{P}_{i}^{a}(x), \operatorname{PP}_{j}^{b}(y)\right\}_{D}=-\frac{\gamma}{2 \alpha_{1}} \int\left[\frac{\delta^{2} W_{0}}{\delta+\omega_{a}^{i}(y) \delta^{+} \omega_{c}^{k}(z)} \frac{\delta \Gamma_{c}^{k}(z)}{\delta E_{m}^{d}(w)} \frac{\delta^{2} W_{0}}{\delta-\omega_{d}^{m}(w) \delta^{+} \omega_{b}^{j}(y)}-\left(\begin{array}{l}
i \\
a
\end{array}\right) \leftrightarrow\left(\begin{array}{l}
j \\
b
\end{array}\right)\right] d z d w, \\
& \left\{-\mathscr{P}_{i}^{a}(x),-\mathscr{P}_{j}^{b}(y)\right\}_{D}=-\frac{\gamma}{2 \alpha_{1}} \int\left[\frac{\delta^{2} W_{0}}{\delta-\omega_{a}^{i}(y) \delta^{+} \omega_{c}^{k}(z)} \frac{\delta \Gamma_{c}^{k}(z)}{\delta E_{m}^{d}(w)} \frac{\delta^{2} W_{0}}{\delta-\omega_{d}^{m}(w) \delta-\omega_{b}^{j}(y)}-\left(\begin{array}{l}
i \\
a
\end{array}\right) \leftrightarrow\left(\begin{array}{l}
j \\
b
\end{array}\right)\right] d z d w, \\
& \left\{-\omega_{a}^{i}(x), \mathscr{P}_{j}^{b}(y)\right\}_{D}=\frac{\gamma}{2 \alpha_{1}} \int\left[\frac{\delta \Gamma_{d}^{m}(z)}{\delta E_{i}^{a}(x)} \frac{\delta^{2} W_{0}}{\delta-\omega_{b}^{j}(y) \delta^{+} \omega_{d}^{m}(z)}\right] d z, \\
& \left\{{ }^{+} \omega_{a}^{i}(x),{ }^{+} \mathscr{P}_{j}^{b}(y)\right\}_{D}=\delta_{a}^{b} \delta_{j}^{i} \delta(x, y)-\frac{\gamma}{2 \alpha_{1}} \int\left[\frac{\delta \Gamma_{d}^{m}(z)}{\delta E_{i}^{a}(x)} \frac{\delta^{2} W_{0}}{\delta+\omega_{b}^{j}(y) \delta^{-} \omega_{d}^{m}(z)}\right] d z,
\end{aligned}
$$

If from now on we use only the Dirac brackets, we are allowed to eliminate all the second class constraints, in particular (46). Thus, writing everything only as functions of ${ }^{+} \omega_{d l}$ and $E^{d l}$ the scalar and vector constraints become

$$
\begin{aligned}
& S=4 \alpha_{1} e^{-1} \epsilon^{i j k} E_{j}^{b} E_{k}^{c}\left[-\gamma^{2} F_{b c i}\left({ }^{+} \omega\right)+\left(\gamma^{2}+1\right) F_{b c i}(\Gamma)\right], \\
& V_{d}=-4 \alpha_{1}\left[\gamma E^{c k} F_{d c k}\left({ }^{+} \omega\right)+\left(\gamma^{2}+1\right) K_{d}^{k}\left[{ }^{+} \mathcal{D}_{c} E^{c}\right]_{k}\right] .
\end{aligned}
$$

The expression of $+\mathscr{P}$ as a function of ${ }^{+} \omega_{d l}$ and $E^{d l}$ is

$$
\begin{aligned}
{ }^{+P_{k}^{d}=4 \alpha_{2} E_{k}^{d}+\epsilon^{d a b}\left[\left(4 \alpha_{4} \gamma-4 \alpha_{3} \gamma^{2}\right) F_{a b k}\left({ }^{+} \omega\right)\right.}+\alpha_{3}\left(1+\gamma^{2}\right) F_{a b k}(\Gamma) \\
\left.+\left(6 \alpha_{3}+2 \alpha_{4} \gamma-4 \frac{\alpha_{4}}{\gamma}\right) \epsilon_{k m n} K_{a}^{m} K_{b}^{n}\right] .
\end{aligned}
$$

\section{WHICH $W_{0}$ LEAD TO CANONICAL TRANSFORMATIONS?}

The Dirac algebra among the basic variables found in the previous section for arbitrary $W_{0}$ is quite complicated. In this section we investigate the possible choices of $W_{0}$ such that $\left(4 \alpha_{1} E / \gamma,{ }^{+} \omega\right) \rightarrow\left({ }^{+} \mathscr{P},{ }^{+} \omega\right)$ is a canonical transformation.

The necessary and sufficient condition that one needs to satisfy is

$$
\int\left[\frac{\delta \Gamma_{d}^{m}(z)}{\delta E_{i}^{a}(x)} \frac{\delta^{2} W_{0}}{\delta+\omega_{b}^{j}(y) \delta-\omega_{d}^{m}(z)}\right] d z=0
$$


for all field configurations and for $W_{0}$ a functional of ${ }^{+} \omega$ and ${ }^{-} \omega$ respectively. This condition holds if and only if

$$
\frac{\delta^{2} W_{0}}{\delta+\omega_{b}^{j}(y) \delta-\omega_{d}^{m}(z)}=0
$$

whose solution is given by $W_{0}\left[{ }^{+} \omega,{ }^{-} \omega\right]=W_{0}^{+}\left[{ }^{+} \omega\right]+W_{0}^{-}\left[{ }^{-} \omega\right]$. We should point out that the integral equation

$$
\int \frac{\delta \Gamma_{d}^{m}(z)}{\delta E_{i}^{a}(x)} V_{m}^{d}(z, y) d z=0
$$

admits non trivial solutions. Recall that each $E_{i}^{a}$ gives a unique spin connections $\Gamma_{a}^{i}$; however, this relationship is not invertible. The reason is that $\Gamma_{a}^{i}(E)=\Gamma_{a}^{i}(\lambda E)$ for $\lambda=$ constant. Therefore, only the scale invariant geometry ( $E$ up to a constant factor) can be recovered from $\Gamma_{a}^{i}$. This implies a non trivial solution of equation (66), for instance $V_{i}^{a}(x, y)=E_{i}^{a}(x) \Omega(y)$. Nevertheless, that solution depends explicitly on $E$ and cannot be realized by derivatives of $W_{0}$. This in turn implies that the canonical transformation takes the simple form

$$
{ }^{+} \mathscr{P}_{i}^{a}=4 \frac{\alpha_{1}}{\gamma} E_{i}^{a}+\frac{\delta W_{0}^{+}}{\delta^{+} \omega_{a}^{i}} .
$$

Recall that we started from the most general action principle for general relativity in the tetrad first order formulation. Therefore, in addition to the factorization property written above, the generating function must derive from a particular combination of the Pontrjagin, Euler and NiehYan invariants (which are Lorentz invariant). The general solution to these constraints is $\gamma= \pm i$ (or equivalently $-2 i \alpha_{1}=2 \alpha_{2}-\alpha_{5}$ ) and $\alpha_{3}$ and $\alpha_{4}$ arbitrary. The canonical transformation (67) becomes in this case

$$
{ }^{+} \mathscr{P}_{i}^{a}=\mp i 4 \alpha_{1} E_{i}^{a}+2\left(\alpha_{3} \pm i \alpha_{4}\right) \epsilon^{a b c} F_{b c i}\left({ }^{+} \omega\right)
$$

which corresponds to the one obtained in [9] for the special case $\alpha_{5}=0$ and $\alpha_{3}=i \alpha_{4}$. For instance when $\alpha_{5}=0$ the action becomes

$$
\begin{aligned}
S[e, \omega] & =\int \alpha_{1}\left(e^{I} \wedge e^{J}\right) \wedge F_{J I}\left(\omega^{S D}\right) \\
& +\left(\alpha_{3}-i \alpha_{4}\right) d \mathscr{L}_{C S}\left(\omega^{A S D}\right)+\left(\alpha_{3}+i \alpha_{4}\right) d \mathscr{L}_{C S}\left(\omega^{S D}\right) .
\end{aligned}
$$

Notice that the momentum shift (68) - analog of the canonical transformation induced by the addition of the Pontrjagin invariant in Yang-Mills theory that introduces the $\theta$ parameter in QCD - can only be obtained for values of the free parameters in the action (2) that make the formulation complex.

\section{THE THETA PARAMETER IN GENERAL RELATIVITY}

In the previous section we have shown that the most general family of connection variables that

can be obtained from the standard Ashtekar-Barbero variables and general action (2) contains the $\theta$ parameter family only in the complex self-dual or anti-self-dual formulations. Therefore, contrary to what one might have naively expected, the real connection formulation of gravity with non trivial $\theta$ is not contained in the family of possible phase space parametrizations stemming from (2). In this section we will show that the requirement of manifest Lorentz invariance, that initially led to (2), is too restrictive to contain that case. 
Indeed one can recover the canonical transformation if one is ready to introduce a boundary term that violates the Lorentz gauge symmetry. More precisely, using the additional structure provided by the gauge condition (19), we define:

$$
\hat{\omega}_{\mu}^{I J}=\epsilon^{I J}{ }_{C D}\left(\gamma \omega_{\mu}^{K C}+\frac{1}{2} \epsilon_{M N}^{C K}{ }_{M N}^{M N}\right) n_{K} n^{D},
$$

Note that $\hat{\omega}$ transforms as an $S O(3)$-connection for $S O(3) \subset S O(3,1)$ gauge transformations that leave invariant the internal vector $n^{I}$. Notice that the components of (70) are such that $\hat{\omega}_{\mu}^{i 0}=0$ for $i=1,2,3$, and $\hat{\omega}_{a}^{i j}=\epsilon^{i j k+} \omega_{a}^{k}$ for $a=1,2,3$ (coordinates adapted to the foliation $\Sigma)$. It follows from this that the curvature components $\hat{F}_{\mu \nu}^{i 0}=0, \hat{F}_{t d}^{i j}=\partial_{[t} \hat{\omega}_{d]}^{i j}-\hat{\omega}_{[t}^{i k} \hat{\omega}_{d]}^{k j}$, and $\hat{F}_{a b}^{i j}=\epsilon^{i j k} F_{a b}^{k}\left[{ }^{+} \omega\right]$. Now we can introduce topological boundary terms defined in terms of the connection (70). Notice that the Euler term vanishes identically. This leaves the Pontrjagin term for $\hat{\omega}_{\mu}^{I J}$ which takes the form

$$
\alpha_{7} \int_{\mathcal{M}} \operatorname{Tr}[F(\hat{\omega}) \wedge F(\hat{\omega})]=2 \alpha_{7} \epsilon^{a b c}\left(\partial_{t}\left({ }^{+} \omega_{a}\right)_{k} F_{b c}^{k}\left({ }^{+} \omega\right)+t^{\mu}\left(\hat{\omega}_{\mu}\right)_{k} D_{a} F_{b c}^{k}\left({ }^{+} \omega\right)\right),
$$

Notice that the second term in the previous expression vanishes due to the Bianchi identities. The Pontrjagin term depends only on ${ }^{+} \omega$; therefore, it satisfies the condition (65). In fact it is obvious from the form of the previous expression that its effect is the expected one producing the canonical transformation

$$
{ }^{+} \mathscr{P}_{i}^{a}=4 \frac{\alpha_{1}}{\gamma} E_{i}^{a}+2 \alpha_{7} \epsilon^{a b c} F_{b c i}\left({ }^{+} \omega\right)
$$

which is real for real Immirzi parameter $\gamma$.

We have just shown how the theta term in quantum gravity can be obtained from the addition of the total derivative (71). In other words, the canonical transformation studied in the quantum context in [12] cannot be obtained from the most general manifestly Lorentz invariant first order formulation of gravity. In order to define the appropriate boundary term one needs to introduce a boundary term that brings in an $S O(3) \subset S L(2, C)$ by explicitly choosing an internal vector $n^{I}$. This might seem strange at first sight as one would seem to be violating both Lorentz invariance in sharp conflict with general covariance. From the point of view of the classical theory is its clear that this is not the case since the term added has no effect on the equation of motion of the theory. However, the situation might appear more obscure in the quantum theory. After all we have seen that - as in QCD - the theta term can have important dynamical as well as kinematical effects in the quantum theory. So even when it is clear that no violation of Lorentz or diffeomorphism invariance is present in the classical theory (i.e. on shell) we need to make sure that this remains true in the context of quantum gravity where off-shell contributions to physical amplitudes cannot be avoided.

So, can the boundary term (71) produce a Lorentz violating effect in the quantum theory? The answer to this question is in the negative as we argue now. The reason is the topological character of (71). The quantity computed in (171) is proportional to the Pontrjagin invariant of an $S U(2)$ principal bundle obtained through the choice of an internal normalized vector $n^{I}$ (for a mathematical description see [14]). As the latter takes discrete values it must be invariant under continuous deformations of $n^{I}$. It remains the question of whether there are homotopically inequivalent choices of $n^{I}$. This correspond to the possible winding of the maps from $\mathscr{M}=\Sigma \times \mathbb{R}$ into the hyperboloid $H \in \mathbb{M}^{4}$ defined by the condition $n^{I} n^{J} \eta_{I J}=-1$. As this winding is trivial we conclude that the term (71) is independent of the choice of $n^{I}$ and hence well defined.

\section{CONCLUSIONS}

We have completed the canonical analysis of the general action (2) and obtained the Dirac bracket for arbitrary values of the couplings $\alpha_{1}$ to $\alpha_{6}$. As long as we restrict to this action the 
family of connection formulations is described by the following two cases:

1. real variables The phase space variables are labelled by an $S U(2)$ connection is given by ${ }^{+} \omega=\Gamma+\gamma \hat{K}$ with Immirzi parameter $\gamma=2 \alpha_{1} /\left(2 \alpha_{2}-\alpha_{5}\right)$ and $\alpha_{3}=\alpha_{4}=0$, and conjugate momentum $+\mathscr{P}_{i}^{a}=4 \frac{\alpha_{1}}{\gamma} E_{i}^{a}$. This shows that both $\alpha_{2}$ and $\alpha_{5}$ enter the definition of the Immirzi parameter. Hence it is possible to obtain a non trivial $\gamma$ by simply adding the Nieh-Yang topological invariant to the Palatini action as shown in [10].

2. complex variables The configuration variable is described by a self dual or antiselfdual connection ${ }^{+} \omega=\Gamma+\gamma \hat{K}$ with $\gamma= \pm i$ and the other parameters constrained to satisfy $\pm i\left(2 \alpha_{2}-\alpha_{5}\right)=2 \alpha_{1}$, with $\alpha_{3}$ and $\alpha_{4}$ arbitrary. The conjugate momentum is ${ }^{+} \mathscr{P}_{i}^{a}=4 \frac{\alpha_{1}}{\gamma} E_{i}^{a}+2\left(\alpha_{3}+\gamma \alpha_{4}\right) \epsilon^{a b c} F_{b c i}\left({ }^{+} \omega\right)$. This second set contains the one studied by [9] as a subclass.

If in turn one wants to describe the effects of $S U(2)$ large gauge transformations for real variables by the addition of a term to the first order action one has no choice but to break manifest Lorentz invariance by the addition of the term (71) to the action (2) with parameters in the first class above. This explicit symmetry breaking is only apparent as the term added does not affect the classical equations of motion on the one hand, and it does change the quantum theory but in a Lorentz invariant way as argued in the last section.

Finally notice that if one is ready to break manifest Lorentz invariance in a more general way then the set of possible connection formulations become infinite dimensional. For instance the canonical transformations

$$
\left({ }^{+} \omega_{a}^{i},{ }^{+} \mathscr{P}_{j}^{b}\right) \rightarrow\left(\tilde{}^{i}{ }_{a}^{i}={ }^{+} \omega_{a}^{i}+\delta W\left[{ }^{+} \mathscr{P}\right] / \delta^{+} \mathscr{P}_{i}^{a},{ }^{+} \mathscr{P}_{j}^{b}\right)
$$

for $W[+\mathscr{P}]$ an arbitrary diffeomorphism invariant and $S U(2)$ invariant functional of ${ }^{+} \mathscr{P}_{a}^{i}$. This kind of canonical transformation - consisting of shifting one canonical variable by the total derivative of a functional of the canonically conjugate one - is available in any field theory. For instance in the case of a real scalar field $\phi$ with conjugate momentum $\pi$ then the analog of the canonical transformations above is given by the shift $(\pi, \phi) \rightarrow(\pi+f(\phi), \phi)$ for some $f: \mathbb{R} \rightarrow \mathbb{R}$. The quantization in this case strongly depends on the choice of canonical variables. Notice that this transformation would turn a simple free theory (which can straightforwardly quantized using for instance the Fock representation) into a highly non linear (depending on $f(\phi)$ ) theory where those techniques cannot be directly applied.

The situation in the case of LQG is much simpler at first sight. The reason is that the canonical transformation (73) preserves the connection nature of the configuration variable and therefore allows for a straightforward implementation of the standard LQG quantization techniques: definition of holonomy-flux algebra of basic kinematical observables and construction of the (unique) diffeomorphism invariant representation. However, this uniqueness of the construction appears to have some unexpected implications. On the one hand questions concerning the geometric interpretation of the kinematical variables in the kinematical Hilbert space seem to arise, as well as the possibility of physically distinguishable sectors (due to the potential unitarily inequivalence of the different formulations). As this concerns entirely the quantum theory these questions will be investigated elsewhere. In the appendix we explicitly exhibit the infinite dimensional nature of this family of connection formulations.

\section{ACKNOWLEDGEMENTS}

The authors would like to thank discussions with M. Montesinos and C. Rovelli on the subject of this work. A.P. acknowledges the support of the Institut Universitaire de France and the grant ANR-06-BLAN-0050 of the Agence Nationale de la Recherche. 


\section{APPENDIX}

In order to simplify the notation let us assume that we are in the real connection variables setting. The functional $W[+\mathscr{P}]$ appearing in (73) can be simply thought of a functional $W[E]$ as the triad $E$ is proportional to $+\mathscr{P}$ (at least in the $\alpha_{7}=0$ case). An example of suitable generating functional is

$$
W_{2}[E]=\int_{\Sigma} \lambda_{1} \mathscr{L}_{C S}(\Gamma)+\lambda_{2} \sqrt{\operatorname{det}(E)}+\lambda_{3} R[E] \sqrt{\operatorname{det}(E)}+\lambda_{4} R_{a b c d} R^{a b c d}[E] \sqrt{\operatorname{det}(E)}+\cdot(
$$

where $R[E]$ and $R_{a b c d}$ is the scalar curvature and Riemann tensor of $\Sigma$ associated to $E$, and $\mathscr{L}_{C S}$ is the Chern-Simons Lagrangean evaluated in the spin connection $\Gamma_{a}^{i}(E)$. Unlike the previous case, generating function $W_{2}$ contains infinitely many parameters - we have given a few characteristic examples; however, any scalar density local functional of $E$ is assumed to be contained in $W_{2}$. The action on the connection variables is to shift the connection as $(A, E) \rightarrow\left(A+\delta W_{3} / \delta E, E\right)$. This observation implies that an infinite dimensional set of simple connection variables for general relativity exist.

Notice the word simple in the previous sentence. The fact that the connection formulations infinitely dimensional should have been expected from the fact that any phase space functional generates a Hamiltonian vector field which (if the latter is diffeomorphism invariant and gauge invariant) can be viewed as a one parameter family of canonical transformations preserving the connection nature of the variables. However, the most general transformation is generated by functional depending on both the connection and the electric field and in general these transformations will not be analytically integrable or be more complicated. Here we concentrate on infinitesimal canonical transformations which can be explicitly exponentiated and lead to a close formula for the new variables as a function of the old ones.

Among the functionals of both the connection $A$ and $E$ whose associated Hamiltonian flow can be integrated in close form there is an important example, namely

$$
W_{3}[A, E]=\epsilon \int_{\Sigma}\left(A_{a}^{i}-\Gamma_{a}^{i}\right) E_{i}^{a}+\cdots
$$

The generating function $W_{2}$ generates rescaling of the Immirzi parameter $\gamma \rightarrow(1+\epsilon) \gamma$. The exponentiated version generates finite redefinition of the Immirzi parameter. This one is clearly not unitarily implementable at the kinematical level in LQG.

The existence of an infinite dimensional set of possible $S U(2)$ connection formulations of general relativity has little interest from the classical point of view. They are all equivalent ways of writing

the same classical theory. However, questions arise as to what the interpretation and effects of these parameters might be in the quantum theory. These questions will be addressed elsewhere.

[1] T. Thiemann, "Modern Canonical Quantum GR," Cambridge, UK: Univ. Pr. (2007). C. Rovelli, "Quantum gravity," Cambridge, UK: Univ. Pr. (2004) 455 p. C. Rovelli, " Quantum gravity," Cambridge, UK: Univ. Pr. (2004) 455 p. A. Ashtekar and J. Lewandowski, "Background independent quantum gravity: A status report," Class. Quant. Grav. 21, R53 (2004) arXiv:gr-qc/0404018. A. Perez, "Introduction to loop quantum gravity and spin foams," Proceedings of the International Conference on Fundamental Interactions, Domingos Martins, Brazil, (2004) arXiv:gr-qc/0409061.

[2] A. Ashtekar, "New Variables for Classical and Quantum Gravity," Phys. Rev. Lett. 57 (1986) 2244.

[3] J. F. Barbero G., "Real Ashtekar variables for Lorentzian signature space times," Phys. Rev. D 51 (1995) 5507 arXiv:gr-qc/9410014.

[4] G. Immirzi, "Real and complex connections for canonical gravity," Class. Quant. Grav. 14 (1997) L177 arXiv:gr-qc/9612030. 
[5] S. Holst, "Barbero's Hamiltonian derived from a generalized Hilbert-Palatini action," Phys. Rev. D 53 (1996) 5966 arXiv:gr-qc/9511026.

[6] N. Barros e Sa, "Hamiltonian analysis of general relativity with the Immirzi parameter," Int. J. Mod. Phys. D 10 (2001) 261 arXiv:gr-qc/0006013.

[7] S. Alexandrov, "Reality conditions for Ashtekar gravity from Lorentz-covariant formulation," Class. Quant. Grav. 23 (2006) 1837 arXiv:gr-qc/0510050. S. Alexandrov, "SO(4,C)-covariant Ashtekar-Barbero gravity and the Immirzi parameter," Class. Quant. Grav. 17 (2000) 4255 arXiv:gr-qc/0005085.

[8] S. Alexandrov, "Hilbert space structure of covariant loop quantum gravity," Phys. Rev. D 66 (2002) 024028 arXiv:gr-qc/0201087]. S. Alexandrov and E. R. Livine, "SU(2) loop quantum gravity seen from covariant theory," Phys. Rev. D 67 (2003) 044009 arXiv:gr-qc/0209105.

[9] M. Montesinos, "Self-dual gravity with topological terms," Class. Quant. Grav. 18 (2001) 1847 arXiv:gr-qc/0104068.

[10] G. Date, R. K. Kaul and S. Sengupta, "Topological Interpretation of Barbero-Immirzi Parameter," arXiv:0811.4496 [gr-qc].

[11] S. Mercuri, "From the Einstein-Cartan to the Ashtekar-Barbero canonical constraints, passing through the Nieh-Yan functional," Phys. Rev. D 77 (2008) 024036

S. Mercuri, "Nieh-Yan invariant and fermions in Ashtekar-Barbero-Immirzi formalism," arXiv:gr-qc/0610026.

S. Mercuri, "Fermions in Ashtekar-Barbero-Immirzi formulation of general relativity," Phys. Rev. D 73 (2006) 084016 arXiv:gr-qc/0601013.

[12] D. J. Rezende and A. Perez, "The theta parameter in loop quantum gravity: effects on quantum geometry and black hole entropy," Phys. Rev. D 78 (2008) 084025 arXiv:0711.3107 [gr-qc]].

[13] A. Ashtekar, A. P. Balachandran and S. Jo, "The CP problem in quantum gravity," Int. J. Mod. Phys. A 4, 1493 (1989).

[14] L. Fatibene, M. Francaviglia and C. Rovelli, "On a covariant formulation of the Barbero-Immirzi connection," Class. Quant. Grav. 24 (2007) 3055 arXiv:gr-qc/0702134. L. Fatibene, M. Francaviglia and C. Rovelli, "Spacetime Lagrangian Formulation of Barbero-Immirzi Gravity," Class. Quant. Grav. 24 (2007) 4207 arXiv:0706.1899 [gr-qc]]. 